\title{
Kalinin II: relato de experiência de um Escape Room aplicado no ambiente educacional
}

\author{
Guilherme S. Costa ${ }^{1}$, Ramon C. Barbosa ${ }^{1}$, Átila M. Costa ${ }^{1}$, Silvia C. Bertagnolli' ${ }^{1}$, \\ Márcia H. Islabão Franco ${ }^{1}$, Marcelo Augusto R. Schmitt ${ }^{1}$, Fábio Y. Okuyama ${ }^{1}$ \\ ${ }^{1}$ Instituto Federal de Educação, Ciência e Tecnologia do Rio Grande do Sul (IFRS) \\ 90.030-041 - Porto Alegre - RS - Brasil \\ \{guilhermescosta, ramonliliane, atila.costa.1988\}@gmail.com, \\ \{silvia.bertagnolli, marcia.franco, marcelo.schmitt, \\ fabio.okuyama\} @poa.ifrs.edu.br
}

\begin{abstract}
This article presents the experience report that used the escape room style of play as a learning strategy. It was applied the EscapED framework to elaborate the game, which systematizes a set of elements that enable the development of this type of game in the educational context. The methodological procedures used were bibliographic research and case study. Data collection was conducted based on an adaptation of the MEEGA+ model, which was used to evaluate the experience. The results identified were promising, which expands the perspective for new studies using this style of play in the educational environment.
\end{abstract}

Resumo. Este artigo apresenta um relato de experiência que utilizou como estratégia de aprendizagem o estilo de jogo escape room. Toda a elaboração do jogo baseou-se no framework EscapED, o qual sistematiza um conjunto de elementos que viabilizam o desenvolvimento deste tipo de jogo no contexto educacional. Os procedimentos metodológicos utilizados foram a pesquisa bibliográfica e estudo de caso. A coleta de dados foi conduzida a partir de uma adaptação do modelo MEEGA+, o qual foi usado para avaliação da experiência. Os resultados, identificados foram promissores, o que amplia a perspectiva para novos estudos utilizando esse estilo de jogo no ambiente educacional.

\section{Introdução}

A aprendizagem baseada em jogos, ou Game-Based Learning (GBL) [Prensky 2001] é uma tendência que vem sendo incorporada cada vez mais na educação, sendo que nos últimos anos, o estilo de jogo Escape Games ou Escape Rooms tem disso utilizado como estratégia pedagógica no contexto educacional. Eles jogos têm como premissa que o jogador precisa escapar (do inglês: Escape) de um lugar explorando uma sala (do inglês: Room), resolvendo pistas que nela estão distribuídas e escondidas [Pereira et al. 2018]. Nesse tipo de jogo o jogador atua integralmente na ação do jogo, e onde os jogadores solucionam enigmas para concluir ações em uma ou mais salas, com o propósito de cumprir um objetivo específico (geralmente, fugir de uma sala) em um tempo limitado [Nicholson 2016]. Uma outra característica que alguns desses jogos utilizam é a interação social para resolver os problemas que se apresentam na sala. De acordo com Nicholson (2016), esse estilo de jogo é ideal para ser aplicado em sala de 
aula, pois combinam uma atividade interpretativa e investigativa lúdica de forma integrada a um determinado conteúdo.

Adicionalmente, Lev Vygotsky e Jean Piaget entendem o jogo é visto como parte importante para o desenvolvimento do aluno, e assim, desenvolveram diversas teorias cognitivistas relacionadas ao lúdico. Vygotsky (1998) destaca aspectos da percepção, atenção, memória, e linguagem, e considera o jogo como ferramenta importante para o desenvolvimento cognitivo, motivacional e social. Corroborando com esta teoria está Piaget (1998), que reconhece a importância dos jogos nos processos de desenvolvimento, para ele o jogo tem uma relação estreita com a construção da inteligência e possui uma efetiva influência como instrumento incentivador e motivador no processo de aprendizagem. Para Vygotsky (1998), as interações sociais são estimuladoras do conhecimento, pois a aprendizagem ocorre quando intermediada pelo outro, onde, a partir da interação social ocorrem a geração de novas experiências e conhecimento. Segundo o autor, a aprendizagem acontece no intervalo da Zona de Desenvolvimento Proximal (ZDP), onde o conhecimento real é aquele que o aluno é capaz de aplicar sozinho e o potencial é aquele que ele precisa do auxílio de outros. Nesse sentido, jogos do estilo escape room favorecem a apropriação do conhecimento através da resolução dos problemas em colaboração com os pares.

Com base nisso, o desenvolvimento deste trabalho adotou um percurso metodológico que iniciou pela pesquisa bibliográfica, que permitiu identificar aspectos teóricos que auxiliaram no desenvolvimento do jogo Kalinin II. Entre os trabalhos localizados encontram-se Utsch, Scárdua e Bragança (2016), Pereira et al. (2018), Nicholson (2016), Voros e Sárkozi (2017), Hermanns et al. (2017) e Clarke et al (2016; 2017) que abordam esse estilo de jogo no contexto educacional. Ao concluir essa investigação por pesquisas já desenvolvidas e utilizadas, partiu-se para um estudo de caso, que compreendeu a aplicação e avaliação do jogo com uma turma de estudantes da disciplina de Tecnologias da Informação e Comunicação do Mestrado Profissional em Informática na Educação, no Instituto Federal de Educação, Ciência e Tecnologia do Rio Grande do Sul.

Neste contexto, para a criação do escape room Kalinin II optou-se por utilizar o framework EscapED [Clarke et al. 2016; Clarke et al. 2017]. Na visão dos autores desse framework, jogos nesse estilo devem ater-se as seguintes fases: definição dos participantes delimitação dos objetivos de aprendizagem; estabelecimento do tema do jogo, onde o enredo deve propiciar o engajamento dos estudantes; construção de enigmas; definição de equipamentos; e a sistematização da avaliação. Nesta última etapa optou-se por utilizar na avaliação o modelo MEEGA+ [Petri 2019], que tem como foco avaliar jogos educacionais digitais e não digitais sob dois pontos de vista: a experiência do jogador, o que é importante para saber se o jogo promove ou não o engajamento; e a percepção da aprendizagem, que indica o quanto o jogo favoreceu ou não a aquisição do conhecimento. Observa-se que, alguns dos itens avaliados foram personalizados para se adequar ao contexto da disciplina e do estilo de jogo selecionado.

\section{Framework EscapED}

O framework EscapED foi sistematizado a partir de um conjunto de seis etapas que possibilitam a criação de jogos no estilo escape room, conforme ilustra a Figura 1. Esse framework fornece um modelo que pode ser adotado ou adaptado para criar soluções 
personalizadas de desse estilo para a área da educação [Clarke et al. 2016]. Neste framework, afirma-se que com relação aos participantes é necessário: (i) compreender informações demográficas e educacionais dos jogadores; (ii) estimar o tempo de duração da experiência, que pode ser curta de quinze (15) minutos ou uma experiência mais longa que dure vários dias; (iii) determinar a dificuldade dos enigmas considerando o perfil de formação dos estudantes; (iv) estabelecer o modo/tipo do jogo, que pode ser baseado em cooperação (jogadores trabalham juntos para resolver os enigmas e escapar da sala) e baseado em competição (jogadores competem para serem os primeiros a resolver os enigmas); e (v) definir a escala: escolha o número de participantes para o qual o jogo se destina.

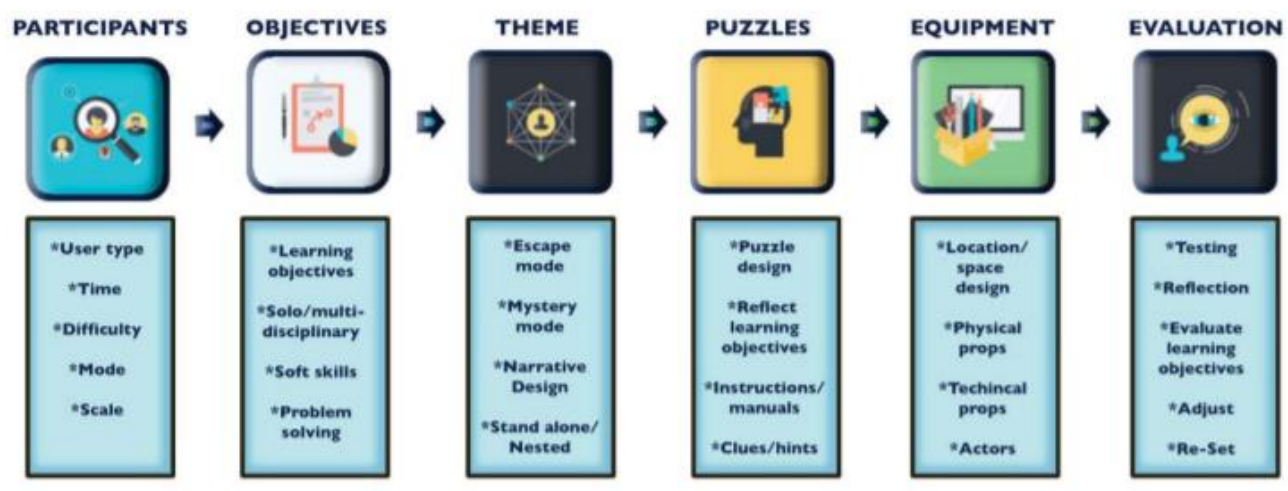

Figura 1. Framework EscapED

Assim, os objetivos de aprendizagem devem ser definidos, pois é através deles que é possível criar um jogo educacional significativo. Eles podem ser abordados no tema, nos enigmas e no enredo do jogo. Ao criar objetivos tangíveis de aprendizagem é possível avaliar a experiência de aprendizagem dos jogadores. O jogo pode ser projetado para que a experiência possa abordar uma disciplina ou várias disciplinas, além disso, nesse framework a sua estrutura deve viabilizar o desenvolvimento de soft skills, tais como comunicação e liderança [Clarke et al. 2016; Clarke et al. 2017]. Outro ponto que está relacionado a esses objetivos diz respeito à solução dos problemas, onde a resolução de enigmas torna a experiência do jogador mais interessante e desafiadora.

Na sequência, o tema, em consonância com o framework EscapED, pode ser desenvolvido de duas formas principais, usando o modo Escape, que tem como foco escapar de uma sala trancada em um horário definido; ou através do modo Mistério, no qual os participantes devem solucionar um mistério em um tempo definido. Em ambos formatos é necessário ter um enredo convincente, de forma que o jogo favoreça o engajamento e o interesse do jogador [Clarke et al. 2016].

No passo seguinte, os enigmas (ou quebra-cabeças) tem como propósito tornar a experiência de jogo interessante, além de personalizar os objetivos de aprendizagem. Para a sua construção é necessário analisar os objetivos e o tema de aprendizagem propostos. As instruções contidas devem ter regras claras e definidas para ajudar e guiar os jogadores na resolução dos problemas que fazem parte do enredo do jogo. Os enigmas podem ainda se apoiar em pistas ou dicas que ajudam o jogador na resolução dos enigmas e ao mesmo tempo garantem a imersão do jogador no jogo [Clarke et al. 2017]. 
Além disso, os equipamentos que devem ser pensados para esse tipo de experiência são [Clarke et al. 2016]: (i) a sala/espaço onde a atividade vai ser conduzida deve ser ampla o suficiente para a experiência do jogo seja confortável, facilitando a movimentação dos jogadores, outro ponto que deve ser observado é que o ambiente deve explorar elementos vinculados ao tema; (ii) suportes físicos: os enigmas e os itens do ambiente devem proporcionar uma experiência realista e viável; (iii) acessórios técnicos: sempre que possível usar tecnologias (computadores, realidade aumenta, realidade virtual, GPS) para aprimorar a experiência do jogo; (iv) atores: se possível usar atores que podem ajudar a concretizar ainda mais a experiência.

Finalmente, a última etapa prevista é a Avaliação que recomenda refletir com os jogadores sobre seus pontos de vista e experiências obtidas na interação com o jogo, de modo a usar o feedback dos jogadores para fazer ajustes na experiência. O framework ainda sugere criar um instrumento de avaliação formal dos objetivos de aprendizagem que foram definidos [Clarke et al. 2017].

\section{Percurso Metodológico}

Conforme já mencionado, o presente trabalho iniciou-se pela pesquisa bibliográfica, onde foram realizadas investigações sobre os aspectos teóricos e teorias de aprendizagem que podem ser aplicadas ao contexto de jogos educacionais. E ainda, foram analisados alguns trabalhos que que abordavam a temática de jogos do estilo escape room.

No caso do trabalho "Festa Estranha" [Utsch, Scárdua and Bragança 2016], o jogo explora a "interação social é necessária para sair da festa", conforme destacam os autores o número de interações é variável para cada estilo de jogador e ela ainda possibilita se chegar a finais diferentes. Já o trabalho de Pereira et al. (2018) concentrou-se em aplicar os princípios da teoria de aprendizagem construtivista para criar um jogo do estilo escape room para ensinar os princípios da lógica de programação a indivíduos que não programavam previamente. Ambos trabalhos, foram desenvolvidos para plataforma digital. Eles apontam a importância de se explorar e planejar as interações sociais nesse estilo de jogo.

Outros trabalhos, que usam estrutura física de escape room são: (i) Nicholson (2016) que realiza uma organização sistematizada de dados obtidos através de uma pesquisa sobre 175 salas do estilo "Escape Room" que foram instaladas ao redor do mundo. Esse trabalho apresentou vários resultados importantes, dentre os quais se destacam: $56 \%$ dos usuários de escape room físicas são adultos ou jovens adultos, exatamente o perfil dos estudantes do curso em que a prática foi aplicado; outro dado apontado é que 24\% representavam um lugar e um tempo específico (entre 1900-2000), esse dado foi determinante para o planejamento da sala proposta; (ii) o jogo escape room físico "Styx - the river" é descrito por Voros e Sárkozi (2017), nele o estudante tem a tarefa de resolver questões relacionadas a física dos fluidos. A sua narrativa envolve elementos fictícios, mas que colocam o estudante no papel de um viajante, percorrendo um mundo pistas e enigmas que os conduzem em sua jornada. A partir desse trabalho foi possível perceber que a inclusão de pistas falsas também poderia ser uma estratégia do Kalinin II; (iii) em Hermanns et al. (2017) a escape room física é usada para auxiliar os alunos no seu processo de aprendizagem sobre medicamentos cardiovasculares, no contexto de um curso de farmacologia. Este trabalho aponta que o 
planejamento do tempo deve ser adequado para evitar frustrações. Logo, ele influenciou na organização do jogo aqui proposto, pois os estudantes foram previamente avisados do tempo disponível e de que esse estilo de jogo possuía restrições temporais; (iv) os trabalhos de Clarke et al $(2016 ; 2017)$ foram essenciais para o desenvolvimento deste trabalho, pois, foi com base no framework proposto, que a maioria dos elementos do Kalinin II foram planejados.

Ao concluir a pesquisa bibliográfica iniciou-se com o projeto do jogo, onde foram aplicados todas os aspectos teóricos apontados nos trabalhos relacionados. Optou-se por utilizar o framework EscapED, pois com base na sua organização o processo de sistematização (projeto, teste e aplicação do jogo) do Kalinin II ficou descomplicado. A seção 3.1 detalha como cada item do EscapED foi adequado ao contexto do jogo Kalinin II, e a seção 3.2 descreve como o jogo foi aplicado e avaliado.

Por fim, foi realizado um estudo de caso que compreendeu a aplicação e a avaliação do jogo com uma turma de estudantes da disciplina de Tecnologias da Informação e Comunicação do Mestrado Profissional em Informática na Educação, no Instituto Federal de Educação, Ciência e Tecnologia do Rio Grande do Sul. No caso dessa disciplina, um dos conteúdos programáticos é como os jogos podem ser aplicados na área da educação. A ideia era permitir aos alunos vivenciar uma experiência de jogo em sala de aula, e que abordasse um estilo ainda pouco utilizado como estratégia pedagógica. Esse estudo de caso possibilitou avaliar o estilo de jogo e fatores ambientais que podem influenciar o seu sucesso. Observa-se que, durante a avaliação foi realizada a coleta de dados usando um questionário com perguntas abertas $\mathrm{e}$ fechadas, o qual possibilitou identificar o nível de conhecimento prévio dos participantes sobre o estilo de jogo adotado, o potencial desse estilo de jogo como ferramenta para auxiliar a aprendizagem, além de itens que avaliam a usabilidade e a experiência do jogador, conforme estabelece o modelo MEEGA+. Esse questionário permitiu, ainda, realizar a coleta dos dados relativos à experiência realizada na sala de aula, sendo que os resultados são detalhados na seção 4 .

\subsection{Percepções do EscapED aplicadas no Kalinin II}

De acordo com as teorias de aprendizagem, a interação social e a colaboração são fundamentais no desenvolvimento e na aprendizagem dos alunos [Piaget 1998]. Diante do pressuposto, na construção do escape room Kalinin II foi realizada uma adaptação do modelo EscapeED, onde o tipo de jogo abordado foi o colaborativo. De acordo com Kenski (2003), a colaboração "pressupõe a realização de atividades de forma coletiva, ou seja, a tarefa de um complementa o trabalho de outros. Todos dependem de todos para a realização das atividades [...]". Seguindo o que estabelece o modelo foi elaborada a Figura 2, que sintetiza as informações essenciais relacionadas aos participantes.

\begin{tabular}{|l|l|}
\hline Item & Descrição \\
\hline Tipo de jogador & Alunos adultos, com pouca experiência em jogos educacionais \\
\hline Tempo & $\begin{array}{l}30 \text { minutos de partida } \\
\text { com alguns minutos adicionais para a apresentação do tema e posteriormente para a } \\
\text { avaliação do jogo }\end{array}$ \\
\hline Grau de dificuldade & Iniciante, devido ao perfil dos participantes \\
\hline Modo/tipo de jogo & $\begin{array}{l}\text { Colaborativo - todos deviam colaborar para conseguir resolver os enigmas e escapar da sala } \\
\text { onde a atividade foi conduzida }\end{array}$ \\
\hline Escala & 9 participantes - para a execução são necessários de 6 a 12 participantes \\
\hline
\end{tabular}

Figura 2. Kalinin II e identificação de seus Participantes 
Como os participantes possuíam conhecimentos diversos, optou-se por criar um ambiente de emergência, forçando um timer de 30 minutos para impor que eles corressem contra o tempo. Desse modo, foi posicionada no ambiente uma luz de emergência e algumas luzes foram apagadas com o objetivo de gerar tensão aos participantes, característica que é encontrada em salas de escape room reais.

Como a maioria dos participantes tinham conhecimentos de matemática optou-se por abordar no jogo o conteúdo de matrizes, o qual permitiu correlacioná-lo com a história elaborada. Todo o enredo do jogo baseou-se em um cenário histórico, recriando o evento em uma usina nuclear soviética após o incidente de Chernobyl (que ocorreu na Usina V. I. Lenin, localizada na cidade de Pripyat, a cerca de $20 \mathrm{~km}$ da cidade de Chernobyl, na extinta União Soviética). A ideia do jogo era que os participantes (no papel de operador chefe e demais operadores da usina) deveriam resolver os enigmas e ajustar as válvulas para que o reator da usina não explodisse. Para tanto, era necessário posicionar, precisamente, as quatro válvulas usando conceitos matemáticos. $\mathrm{O}$ jogo abordou o conceito de matriz quadrada, ou seja, uma matriz com o mesmo número de linhas e colunas, explorando, ainda, conceitos sobre o funcionamento de usinas nucleares e química. A Figura 3 ilustra o enredo utilizado para engajar os participantes.

A nossa história começa com os preparativos para a ligação da usina de KALiNiN ii na rede elétrica soviética em Fevereiro de 1987. A inauguração da nova usina demandou a necessidade de levar novos operadores para lá. $O$ treinamento da usina KALiNiN ii foi realizado observando os operadores de Kalinin I, porém com os eventos de Chernobyl o sistema de segurança de KALiNiN ii foi modificado para ser simplificado num simples sistema de válvulas reguladas por computadores ao invés de terminais analógicos.

No dia 3 de Fevereiro, os primeiros operadores novatos foram inseridos no controle de Kalinin II e todos os testes ocorreram sem problemas. Eles foram realizados pelo operador chefe, Gennady Kozlov, experiente operador e fanático por anotações. Gennady, preocupado com a situação pré-inauguração da usina levantou muitas anotações sobre questões básicas do seu funcionamento. Essas anotações devem se tornar um guia para que os demais operadores possam entender o funcionamento do sistema. No dia 15 de Fevereiro, durante um dos testes de força, Ivan foi encontrado bebendo Vodka escondido e enquanto foi levado para dar explicações, acabou derrubando as todas as anotações de Gennady pela sala. Ao final do turno Sergei, outro operador reorganizou a sala, porém, as anotações pessoais de outros operadores acabaram se misturando as de Gennady.

Durante as operações diárias do dia 16 de Fevereiro, ocorreu uma emergência: as válvulas do sistema de resfriamento foram alinhadas de forma incorreta na noite anterior causando superaquecimento no reator. Ao ligar o sistema de testes os operadores do turno se surpreenderam com o nível de pressão que ligou o sistema de segurança da usina.

O sistema possui um timer que indica quanto tempo levará para entrar em modo de derretimento do núcleo. O reator VVER ou WWER (do russo: Vodo-Vodyanoi Energetichesky Reaktor; Água-água Energético reator) é composto por quatro válvulas de água pressurizada que resfriam o núcleo, os operadores devem utilizar o terminal e ligar as válvulas corretas para liberar a pressão e iniciar o resfriamento do núcleo.

\section{Figura 3. Kalinin II e o Enredo}

Conforme já mencionado, o jogo se passa em uma usina nuclear soviética, na década de 1980, e a tarefa dos jogadores é utilizar quatro sequências numéricas organizadas no formato de matrizes. Usar uma matriz incorreta acarreta o término do jogo, logo os jogadores devem vasculhar a área designada para encontrar as dicas e anotações dos operadores que estão dispersas pelo cenário. Elas dicas são padronizadas no formato de notas escritas pelos personagens vinculados à usina, podendo apresentar dicas úteis ou apenas textos aleatórios para tirar o foco dos jogadores. Lembrando que o jogo é delimitado por um timer de 30 minutos e que as ações individuais para buscar ou não o significado das notas pode custar tempo para a equipe, cabe ao operador chefe organizar as buscas pelos enigmas no cenário e a operação do terminal. A mecânica desenvolvida é baseada em escape rooms tradicionais, envolvendo um dispositivo de fuga que quando aberto encerra o jogo. A área escolhida para a montagem da experiência do jogo era composta por duas salas interligadas, uma onde estavam distribuídos/escondidos os enigmas em quadros, móveis, estantes e caixas de papel, e a outra onde estava o computador/terminal usado como dispositivo de fuga. 
Além do operador chefe a equipe de operadores conta com uma equipe de apoio, ou Game Masters (GM), que são membros da equipe que auxiliam com dúvidas sobre as regras e solucionam problemas que podem ocorrer em relação às mecânicas do jogo. Os GM participaram como observadores e "motivadores", e seu objetivo era incentivar a busca das dicas e tirar dúvidas sobre as regras do jogo. Assim, a cada 5 minutos eles avisavam o tempo que faltava para o fim do jogo, visando manter os participantes com a atenção focada, e destacando as consequências hipotéticas se ocorresse alguma falha na resolução do problema.

No jogo Kalinin II, foram criados dois tipos de equipamentos, as anotações de papel com informações sobre o cenário (por exemplo, Figura 4) e as dicas de como solucionar o dispositivo de fuga. As anotações apresentam um título sequencial que funciona como um indexador (não existindo dois títulos iguais) para auxiliar os jogadores. Além do título, é apresentado um texto curto, geralmente, em primeira pessoa, que descreve uma ação que o personagem deve realizar. A anotação "Segunda Válvula" (Figura 4) apresenta uma informação importante para o progresso dos jogadores, além de descrever alguns detalhes históricos e científicos sobre a usina do jogo. Embora fossem quatro válvulas, o número de dicas era bem maior, pois algumas indicavam a posição real com o valor a ser utilizado no sistema de válvulas, já outras serviam apenas para distrair e criar frustração, pois essa é uma característica desse estilo de jogo [Voros and Sárkozi 2017].

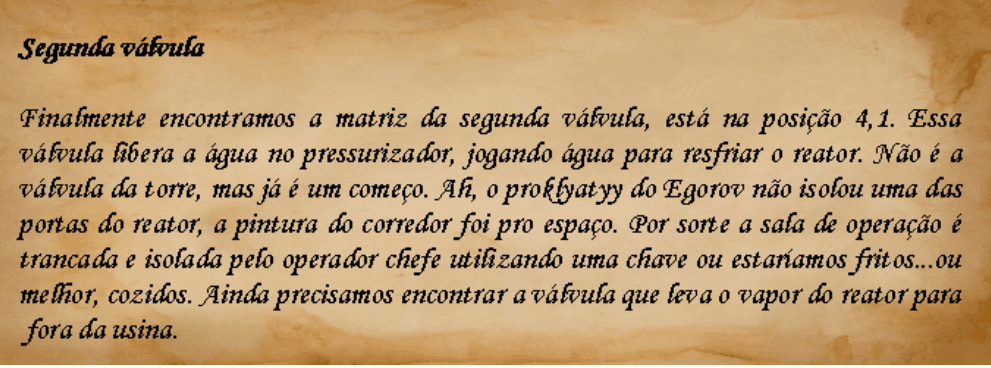

Figura 4. Kalinin II: um exemplo de equipamento

Outro equipamento desenvolvido foi o dispositivo de fuga, um software que simula um terminal de computador dos anos 1980 (Figura 5). Esse terminal apresenta um timer do tempo restante de jogo, um botão para finalizar o jogo e uma área que representa uma tela de um terminal. Esse terminal apresenta quatro áreas de texto (retângulo de destaque na Figura 5), onde os jogadores deveriam substituir os valores de $\mathrm{X}$ e Y de cada uma das válvulas pelos valores encontrados nas anotações.

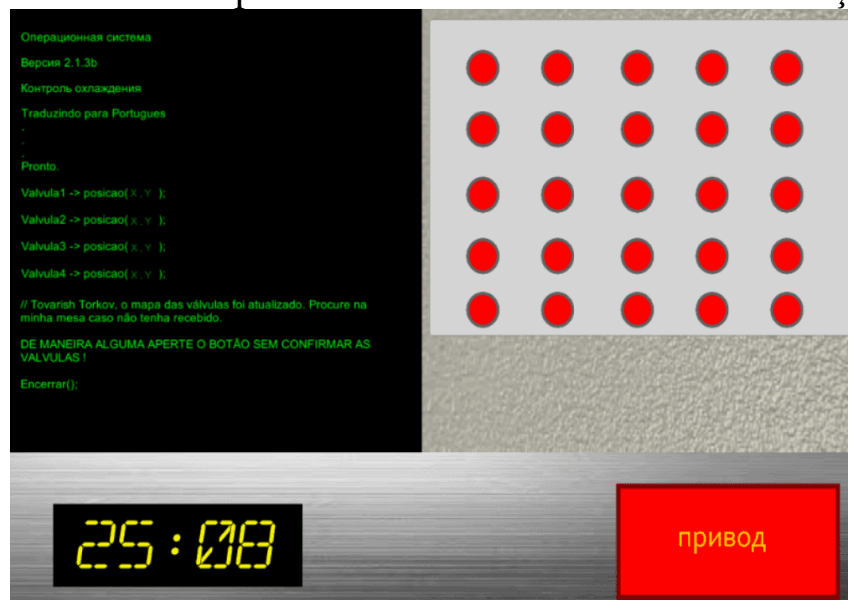

Figura 5. Kalinin II: o dispositivo de fuga 
IX Congresso Brasileiro de Informática na Educação (CBIE 2020)

Anais do XXXI Simpósio Brasileiro de Informática na Educação (SBIE 2020)

\subsection{A condução do estudo de caso}

O estudo de caso iniciou pela apresentação do tema e de como a atividade seria conduzida. Foi explicado como funciona um jogo no estilo escape room para todos os participantes, e que os enigmas, que deveriam ser resolvidos, estavam "ocultos" na sala principal preparada para a experiência. Como a colaboração é essencial para o jogo projetado foi explicitado aos participantes que eles deveriam realizar as atividades em conjunto, e que o grande objetivo seria solucionar o problema de posicionamento das válvulas da usina Kalinin II. Em seguida os doze (12) participantes foram organizados da seguinte forma: nove participantes constituíram uma única equipe, onde um deles foi designado como operador chefe (Gennady) e os outros três participantes atuaram na equipe de apoio. Assim, eles saíram pela sala procurando os enigmas que resolveriam o problema. À medida que os encontravam percebiam que para resolvê-lo seria necessário observar o título sequencial. Eles trocaram ideias entre si, questionaram se os enigmas faziam sentido e perceberam que alguns deles eram apenas para conduzir ao caminho errado. Muitas vezes pediram ajuda à equipe de apoio que participou mais observando $\mathrm{e}$ incentivando os participantes a concluírem a tarefa dentro do tempo delimitado. Enfim, os participantes conseguiram solucionar o problema ajustando as válvulas e evitando que uma nova catástrofe ocorresse no cenário hipotético. Por fim, foi realizada a avaliação do jogo, onde todos refletiram sobre a experiência e apontaram os pontos positivos e o que poderia ser aprimorado. Além disso, eles responderam a um questionário composto por questões abertas e fechadas, o qual permitiu avaliar o jogo como um todo.

\section{Resultados e Discussões}

Conforme já mencionado, o jogo foi avaliado utilizando-se a maioria das questões que integram o modelo MEEGA+, o qual foi utilizado para verificar a compreensão do conteúdo do jogo, além de servir como feedback dos jogadores em relação a atividade, tanto no ponto de vista educacional quanto de projeto. A primeira questão do questionário abordava diretamente se os participantes da experiência conheciam o estilo de jogo escape room, sendo que $62,5 \%$ já conheciam esse estilo, mas nunca haviam experimentado esse tipo de dinâmica no ambiente educacional, sendo que 37,5\% dos participantes apontou que a sua primeira experiência com esse estilo foi o Kalini II. Outro questionamento realizado foi se a prática, utilizando o escape room, auxiliou na construção conceitual sobre algum tipo de conhecimento. Neste caso, todos os participantes apontaram que houve a apropriação de conhecimentos na área da Matemática, Química e História, além de outras questões como trabalho em equipe e raciocínio lógico. Como essa era uma pergunta aberta, os participantes apontaram: Participante 1: "o jogo é versátil tendo a possibilidade de trabalhar diversos campos do conhecimento." e Participante 3: A prática proporcionou a integração e o trabalho em grupo, buscando uma análise da necessidade de interagirmos com o colega para a resolução de problemas.

A Figura 6 ilustra parte dos resultados obtidos com a aplicação dos itens do modelo MEEGA+, considerando os nove participantes que atuaram durante toda a prática pedagógica. As respostas estão organizadas em cores usando a escala Likert definida pelo modelo. Observa-se que o ponto que apresentou maior divergência nas respostas diz respeito à atenção focada. Acredita-se que ela pode ter ocorrido devido ao fato de que o conteúdo foi apresentado através de uma pequena revisão de cinco (5) 
minutos, dessa forma, talvez parte dos participantes não lembrassem o que era uma matriz. Identificou-se que dois itens tiveram avaliações muito positivas, sendo estes, a interação social - visto que os alunos perceberam que se não atuassem de forma conjunta não conseguiriam resolver os enigmas e a percepção da aprendizagem - os participantes apontaram que conseguiram aprender conteúdos vinculados às áreas de história, química e matemática, e destacaram que o uso de jogos pode ser uma estratégia pedagógica pode desenvolver a aprendizagem de forma interdisciplinar, como relata o Participante 6: "Achei uma dinâmica que traz motivação ao grupo para trabalharem em conjunto, buscando e interpretando pistas para resolver um problema. Penso que é possível trabalhar uma variedade de assuntos, inclusive de forma interdisciplinar através dessa abordagem.". Com relação à Usabilidade o que foi mais impactante foi o cenário construído para a realização do jogo, o Participante 8 relatou: "A maneira como foi construído o cenário, as imagens e sons, faziam com que nós tivéssemos a sensação de que realmente estávamos em uma usina nuclear. Numa situação real acredito que todos nós entraríamos em pânico, mas na atividade conseguirmos desenvolver a prática proposta.”. Esse depoimento destaca a importância de se planejar o ambiente e como esse estilo de jogo pode contribuir para o desenvolvimento da habilidade de trabalho em equipe.

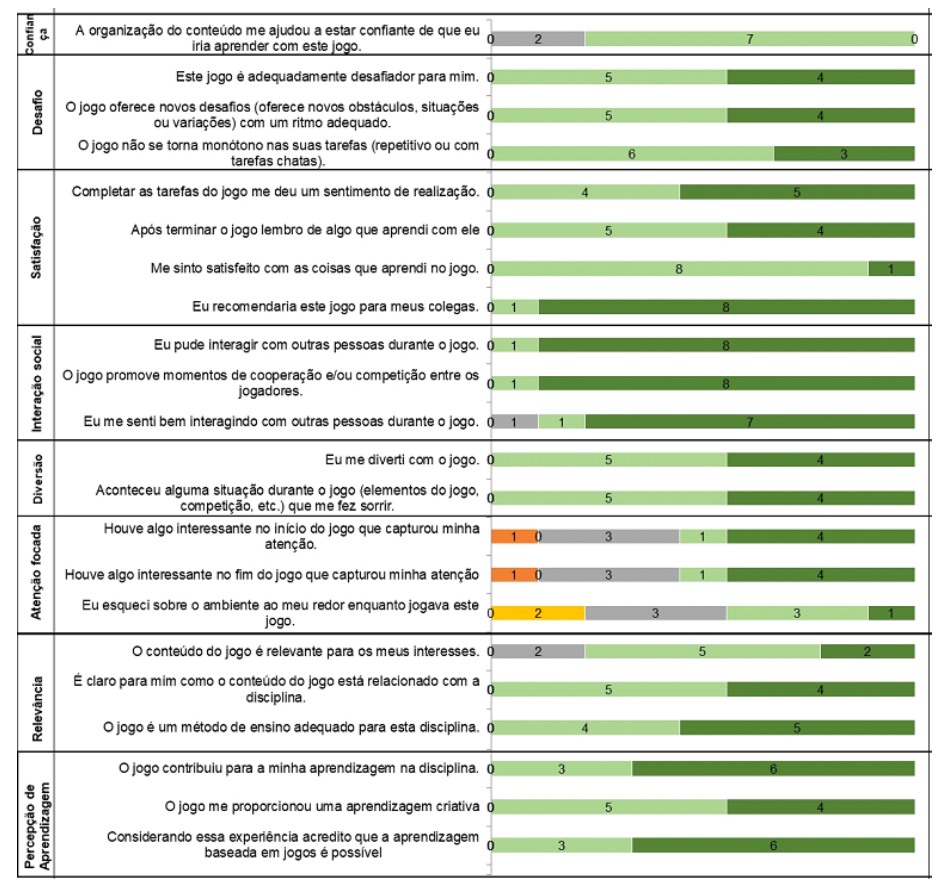

Figura 6. Kalinin II: avaliação modelo MEEGA+

\section{Conclusões}

O trabalho apresentou o uso do framework EscapED aplicado no contexto educacional, com o propósito de propiciar aos participantes vivenciar uma experiência real de jogo no estilo escape room. Durante o processo de desenvolvimento do jogo percebeu-se que esse tipo de jogo pode ser mais simples do que o desenvolvimento de outros tipos de jogos, em especial jogos digitais, pois não exige que a equipe possua desenvolvedores com conhecimentos especializados. Porém, ele exige que a experiência seja conduzida em um ambiente que reflita a realidade do enredo definido. Outra vantagem observada é a simplicidade de adaptar o jogo, usando um mesmo enredo, sendo necessário alterar apenas o objetivo de aprendizagem e customizando os seus enigmas. 
Através dos resultados obtidos observa-se que o jogo proporcionou uma aprendizagem colaborativa, além de possibilitar o desenvolvimento individual, coletivo e acadêmico dos participantes. Dessa forma, contemplando a visão de Vygotsky (1998), de que a aprendizagem é construída pelas interações sociais do sujeito com os outros, sendo que as interações sociais são as principais desencadeadoras do aprendizado. Além disso, colocou-se em prática a GBL, onde foi possível desenvolver a autonomia dos participantes, estimular a tomada de decisões e trabalhar processos de reflexão, integração e reelaboração de novas práticas, características essas consideradas essenciais por Morán (2015).

\section{Referências}

Clarke, S., Pell, D., Arnab, S., Morini, L., Keegan, H., Wood, O. (2017) “EscapED: A Framework for Creating Educational Escape Rooms and Interactive Games to For Higher/Further Education.” International Journal of Serious Games, v. 4, n. 3.

Clarke, S.; Arnab, S.; Morini, L.; Wood, O.; Green, K.; Masters, A.; Bourazeri, A. (2016) "EscapED: A Framework for Creating Live-Action, Interactive Games for Higher/Further Education Learning and Soft Skills Development", Proceedings... European Conference on Games Based Learning, p. 968-672.

Hermanns, M.; Deal, B.; Ann M.; Hillhouse, S.; Opella, B.; Faigle, C.; Campbell, R. (2017) "Using an "Escape Room toolbox approach to enhance pharmacology education". Nursing Faculty Publications and Presentations. v. 8, n. 4.

Kenski, V. M. (2003) “Tecnologias e ensino presencial e a distância”, Papirus.

Morán, J. (2015) "Mudando a educação com metodologias ativas", http://www2.eca.usp.br/moran/wp-content/uploads/2013/12/mudando_moran.pdf>

Nicholson, S. (2016) "Peeking behind the locked door: A survey of escape room facilities", Meaningful Play, http://scottnicholson.com/pubs/stateofescape.pdf.

Pereira, L. T.; Silva, F. H. C.; Palomino, P. T.; Isotani, S. (2018) "A abordagem construtivista no desenvolvimento de um serious game do gênero escape room", SBGames, p. 1011-1018.

Piaget, J. (1998) “O espírito de solidariedade na criança e a colaboração internacional”, in: Sobre a pedagogia - textos inéditos. Casa do Psicólogo, p.59-78.

Prensky M. (2001) “Digital Game-Based Learning”, McGraw-Hill.

Petri, G.; Gresse, W. C. and Borgatto, A. F. (2019) "MEEGA+: Um Modelo para a Avaliação de Jogos Educacionais para o ensino de Computação", In: Revista Brasileira de Informática na Educação (RBIE), 2019.

Utsch, S.; Scárdua, M.; Bragança, L. (2016) "Festa Estranha: Um Estudo de Caso no Desenvolvimento de Indie Games em Grupos Minoritários", Proceedings... SBGames, p. 1234-1236.

Voros, A.; Sárkozi, Z. (2017) "Physics escape room as an educational tool". AIP Conference Proceedings 1916, https://aip.scitation.org/doi/pdf/10.1063/1.5017455

Vygotsky, L. S. (1998) "A formação social da mente: o desenvolvimento dos processos psicológicos superiores”, 6. ed., Martins Fontes. 